\title{
Matrix metalloproteinases and peripheral arterial disease
}

\author{
Chiara Busti · Emanuela Falcinelli · \\ Stefania Momi · Paolo Gresele
}

Published online: 24 September 2009

(c) SIMI 2009

\section{Erratum to: Intern Emerg Med DOI 10.1007/s11739-009-0283-y}

Unfortunately, the two sentences under the sections "Classification, structure, and regulation" (ninth paragraph: The activation of a pro-MMP is the...) and "MMPs and diabetes" (second paragraph: In fact, an upregulation of MMP-2 and -9 in the human...) in the present form are wrong. Please find the correct sentences here.

"The principal way for the activation of pro-MMPs is by the removal of its propeptide domain by tissue or plasmatic proteinases, including active MMPs. Moreover, pro-MMP activation can occur at the surface of cells, as in the case of MMP-2, or at intracellular level, as in the case of MMP-11, $-21,-23,-27$ and MT-MMPs."

"In fact, an upregulation of MMP-2 and -9, which induce the expression of angiostatin, has been shown in the arterial vasculature of diabetics, suggesting that the enhanced production of angiostatin, a potent anti-angiogenic factor that antagonizes the expression and the activity of VEGF [61, 62], may explain the impaired angiogenesis of diabetes [63]."

The online version of the original article can be found under doi:10.1007/s11739-009-0283-y.

C. Busti · E. Falcinelli · S. Momi · P. Gresele $(\square)$ Division of Internal and Cardiovascular Medicine, Department of Internal Medicine, University of Perugia,

Via E. dal Pozzo, 06126 Perugia, Italy

e-mail: grespa@unipg.it 